\title{
Developing a fuzzy model for Tehran's air quality
}

\author{
Nafiseh Tokhmehchi ${ }^{a^{*}}$ and Ahmad Makui ${ }^{\mathrm{b}}$
}

${ }^{a}$ Depatment of Industrial Engineering, Science and Research Branch, Islamic Azad university, Tehran, Iran ${ }^{b}$ Depatment of Industrial Engineering, Iran University of Science and Technology, Tehran, Iran CHRON I C L E AB T R A T

\begin{tabular}{l}
\hline Article history: \\
Received March 18, 2014 \\
Accepted 2 December 2014 \\
Available online \\
December 22014 \\
\hline Air pollution \\
Air Quality monitoring \\
Fuzzy logic
\end{tabular}

\begin{abstract}
This research aims to offer a fuzzy approach for calculating Tehran's air pollution index. The method is based on fuzzy analysis model, and uses the information about air quality index (AQI), included on the website of Tehran's Air Quality Monitoring And Supervision Bureau. The contrived fuzzy logic is considered a powerful tool for demonstrating the information associated with uncertainty. In the end, several graphs visualize this inferential system in various levels of pollution.
\end{abstract}

\section{Introduction}

Air is one of the 5 essential elements for humans to stay alive. Each person breathes a daily average of 22,000 times, and requires about 15 kilograms of air every day. Human can stay alive for 5 weeks without food and 5 days without water, but not even 5 minutes without air. Industrial developments and technological advancements have introduced lots of achievements for human's life, but unfortunately over the course of industrialization trends, various wastes and unwanted materials are released in the environment that are mostly harmful. Industrial pollution affects the environment, significantly. Air pollution phenomenon is another result of industrial development. The increasing population, urbanization, and fossil energy consumption intensifies air pollution every day. Air quality is highly associated with the quality of human's life and respiration. Just like the wind and weather conditions that change every day and every hour, air quality can be oscillating as well. Air Quality Monitoring And Supervision Bureau converts air-quality data in mega-cities into air-quality index (AQI) (Kyrkilis et al., 2007; Gurjar et al., 2008); hence, AQI is a key index for understanding airquality and its relation with human's health. AQI is a general index for daily prediction of air quality. This index informs people about air quality, being clean or polluted, and associates air quality with human's health. In other words, AQI demonstrates the level of air-pollution effect on human's health, and lets the general public have a better understanding of the fact (Tehran's Air Quality Report, 2013).

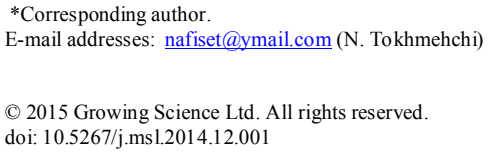




\subsection{Air Quality Index (AQI)}

Air Quality Index (AQI) categorizes air quality into 6 classes. Each class correlates with a particular human's health level. The 6 classes are as follows:

Good: AQI is between 0 and 50. Air quality is satisfactory and air pollution is harmless or low-harm.

Average: AQI is between 51 and 100. Air quality is acceptable, although some pollutants might be harmful to a few people with certain health conditions. For example the ones who are allergic to ozone, might display respiratory symptoms.

Unhealthy For Allergic Groups: AQI is between 101 and 150. Some allergic people might experience certain health complications. For instance the ones with pulmonary diseases are more than regular people in risk, when being exposed to ozone. People with cardiovascular illness will be in risk when exposed to air particles. Apart from special groups, this level of air pollution does not influence on the general public.

Unhealthy: AQI is between 151 and 200. Everybody might experience the health effects of air pollution. Allergic groups are in higher risk, and suffer more intensely from air pollution.

Highly Unhealthy: AQI is between 201 and 300. This condition is alerting. It shall influence severely on everybody's health.

Dangerous: AQI goes beyond 300. This is serious alerting condition for human's health, and is considered an emergency situation. All the society members will be affected in such environment.

\subsection{Calculation of AQI For A Society}

Air quality is measured by determining the thickness of main pollutants in measurement stations. There might be numerous stations based on the population and vastness of the city. The raw data obtained from pollutant measurement will be converted to AQI using Eq. 1. The parameters of this equation are taken from AQI Breakpoints:

$$
I_{p}=\frac{I_{H i}-I_{L o}}{B P_{H i}-B P_{L o}}\left(C_{p}-B P_{L o}\right)+I_{L o}
$$

where $I_{p}$ represents AQI for the pollutant $p, C_{p}$ is the measured thickness of the pollutant $p, B P_{H i}$ stands for the breakpoint with $B P_{H i} \geq C_{p}$. In addition, $B P_{L o}$ is the breakpoint with $B P_{H i}<C_{p}, L_{H i}$ is the amount of AQI according to $B P_{L o}$.

\section{The influential factors in air quality}

US Environmental Protection Agency (EPA) introduces 6 main pollutants as criteria, and divides them into two categories: Primary and secondary. Primary pollutants are released directly in large amount from the sources and lead to negative health effects and well-being problems in relatively large scale. They influence on vast numbers of people in a wide area. National standards have been defined for these pollutants. This category includes 5 elements: Carbon monoxide $(\mathrm{CO})$, nitrogen dioxide $\left(\mathrm{NO}_{2}\right)$, air particles $(\mathrm{PM})$, sulfur dioxide $\left(\mathrm{SO}_{2}\right)$, and lead $(\mathrm{PB})$. Secondary pollutants are created owing to the reactions in atmosphere. The main pollutant of this group is Ozone $\left(\mathrm{O}_{3}\right)$. 


\subsection{Carbon Monoxide}

Carbon monoxide is a kind of colorless, odorless and tasteless gas. It is one of the most abundant pollutants in atmosphere. This gas is commonly the result of imperfect combustion of fossil fuel types. When its level goes beyond the healthy stage, it causes bewilderment and headache initially. In the end it might be lethal. In urban regions, carbon monoxide level is associated with traffic load, and varies in different wind and weather conditions.

\subsection{Nitrogen Dioxide}

There are numerous kinds of nitrogen oxide, but nitrogen dioxide is commonly measured for studying air pollution. Nitrogen oxide types are also the main base of ozone and acid rains, which leave negative effects on water and plant ecosystems. They harm the plants significantly.

\subsection{Air Particles}

Any kind of microscopic or Nano material, except solid and liquid types of pure water, that is detected in air and has bigger size than molecules is called "Particle". The origins of this pollutant include all sorts of combustive reactions, industrial activities, road construction operations, road traffic, and other natural resources such as volcanic activities, jungle fires, wind and dust. Being exposed to air particles leads to short-term effects such as burning of eyes, throat and nose, as well as asthma.

\subsection{Sulfur Dioxide}

Several types of fossil fuel such as coal and heavy petrochemical products have high sulfur content. This sulfur turns into sulfur dioxide gas or air particles after igniting. Power plants, factories, diesel cars and some residential areas that consume such sorts of fuel are the main resources of this pollutant. Likewise nitrogen dioxide, this gas affects and destroys plants.

\subsection{Ozone}

Ozone is formed in atmosphere due to photochemical reactions between the hydrocarbons exhausted from cars and nitrogen oxides. It irritates eyes, throat and lungs. This pollutant is not released from a particular source, but is created in result of the reactions between atmosphere pollutants; therefore, this pollutant is regarded as secondary type. Ozone is a highly reacting oxidant, whose chemical hyperactivity causes various complications including degeneration and disorders in pulmonary tissues.

\section{Designing a fuzzy inference system for measuring Tehran's Air Quality}

Definition: Being fuzzy means having multi-value; i.e. every question has a range of answers (Zadeh, 1965, 1968, 1997, 1982, 1983, 1996).

Definition of Fuzzy Sets and Fuzzification: This study defines the fuzzy sets in compliance with objectives of this research for designing the fuzzy inference system. 5 main factors including quality of carbon monoxide, quality of nitrogen dioxide, quality of air particles, quality of sulfur dioxide and quality of ozone, are the key factors that define the quality of breathing air. These factors are regarded as fuzzy sets. Knowing that, air quality measurement will be based upon those factors. Fig. 1 shows an overall view of the fuzzy inference system in this study (Mamdani, 1974, 1975, 1976, 1977). 


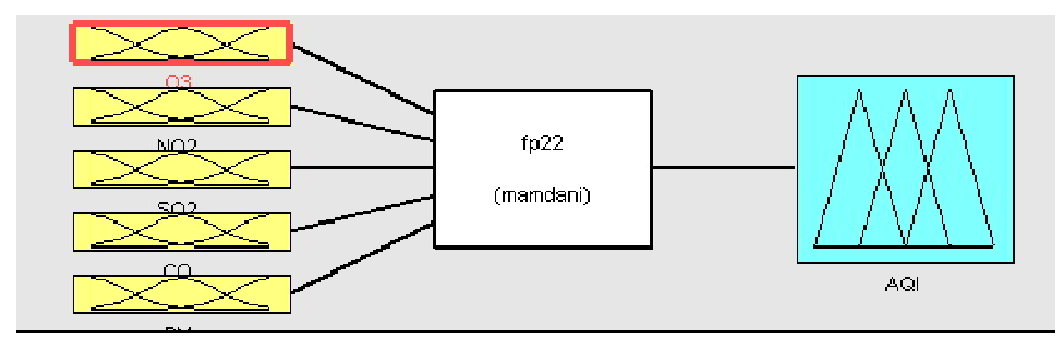

Fig 1. Diagram of fuzzy inference system of air quality

Yellow graphs (left side) are the membership functions of the 5 fuzzy sets, being used as the input of inferential system. The white diagram (in the middle) shows the rules and regulations of analysis, used to analyze the system and process the inputs into outputs. The blue graphs (at the right side) also demonstrate respiratory air quality. In order to define the membership functions of the fuzzy inferential system in this research, the two popular triangular and trapezoidal functions have been applied. Each of the input membership functions have been scaled into 6 qualitative levels: Good, relatively good, relatively bad, very bad, and dangerous. The system's output represents quality of respiratory air. It is scaled with 6 verbal scales: Perfect, regular, unhealthy for the allergic population, unhealthy, highly unhealthy, and dangerous. The following diagrams show the membership functions for each and every input. If ozone quality is good, nitrogen dioxide quality is good, sulfur dioxide quality is good, carbon monoxide quality is good, and air particles quality is also good, then air quality is perfect.

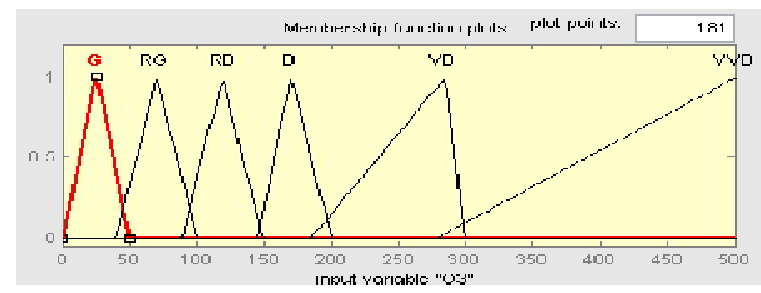

Fig. 2. Diagram of ozone membership function

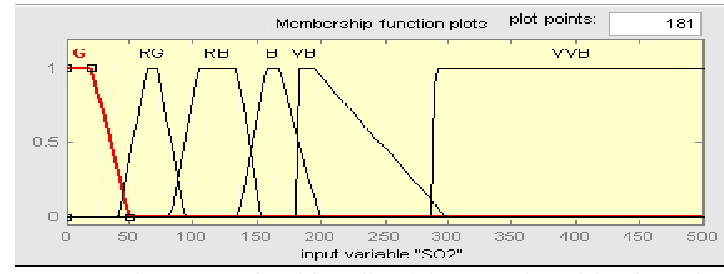

Fig. 4. Diagram of sulfur dioxide membership function

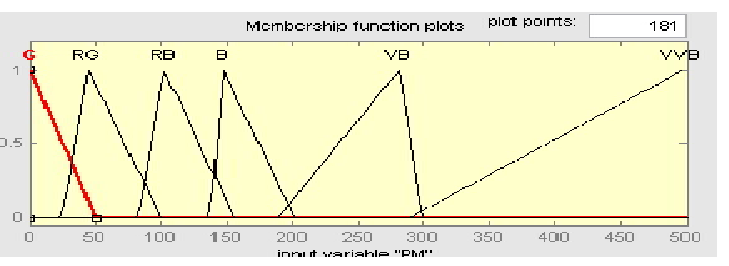

Fig. 6. Diagram of air particles membership function

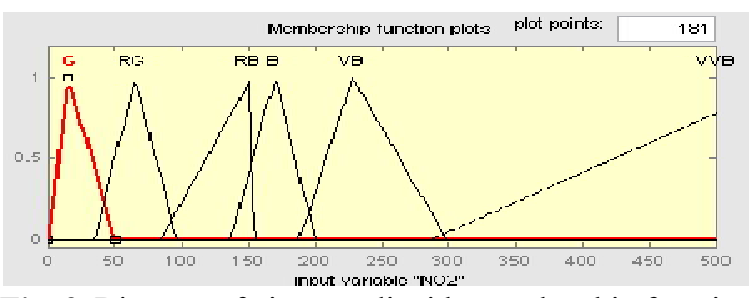

Fig. 3. Diagram of nitrogen dioxide membership function

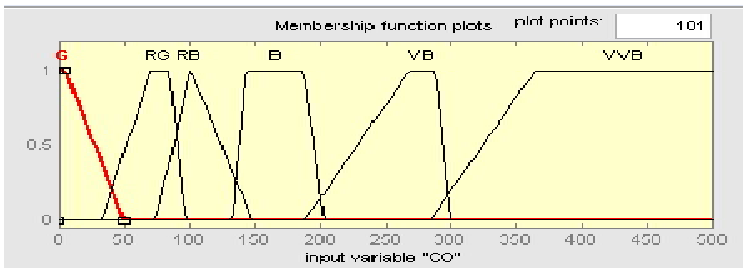

Fig. 5. Diagram of carbon monoxide membership function

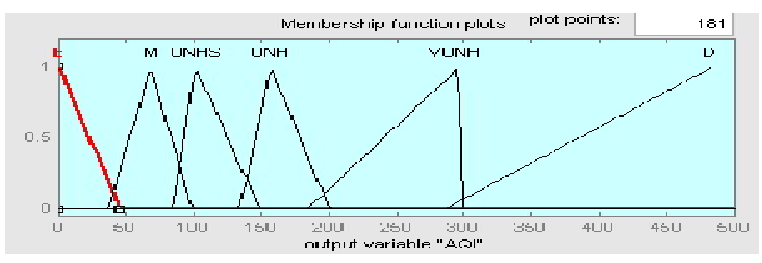

Fig. 7. Diagram of air quality membership function

\section{Defining the rules of fuzzy analysis}

In order to complete the fuzzy inferential system of this study, the rules of fuzzy logics, which are in fact the heart and soul of fuzzy system, shall be defined. These rules present the relation between the defined fuzzy sets in the inferential system with each other, in common with their effects on respiratory air quality. In other words, the input data of fuzzy inferential system are transformed into output data via these rules. The following table displays the fuzzy rules concluded with the help of Air Quality 
Supervision Bureau. The rules defined in Table 1 are processed using "Rules processing" menu in Matlab software. The conclusions of the fuzzy inferential system in this research are shown in Fig. 8. This diagram visualizes the whole fuzzy inferential system in the study. Each row in the diagram represents a fuzzy rule.

\section{Table 1}

25 different rules for air quality

1 If ozone quality is good, nitrogen dioxide quality is good, sulfur dioxide quality is good, carbon monoxide quality is good, and air particles quality is also good, Then air quality is Perfect.

2 If ozone quality is good, nitrogen dioxide quality is relatively good, sulfur dioxide quality is good, carbon monoxide quality is good, and air particles quality is also good, Then air quality is regular.

3 If ozone quality is good, nitrogen dioxide quality is relatively good, sulfur dioxide quality is relatively good, carbon monoxide quality is good, and air particles quality is also good, Then air quality is regular.

4 If ozone quality is good, nitrogen dioxide quality is good, sulfur dioxide quality is good, carbon monoxide quality is relatively good, and air particles quality is also good, Then air quality is unhealthy for the allergic population.

5 If ozone quality is good, nitrogen dioxide quality is good, sulfur dioxide quality is very bad, carbon monoxide quality is good, and air particles quality is also good, Then air quality is unhealthy.

6 If ozone quality is relatively good, nitrogen dioxide quality is bad, sulfur dioxide quality is good, carbon monoxide quality is good, and air particles quality is also good, Then air quality is unhealthy.

7 If ozone quality is relatively good, nitrogen dioxide quality is relatively good, sulfur dioxide quality is good, carbon monoxide quality is good, and air particles quality is also good, Then air quality is allergic population.

8 If ozone quality is good, nitrogen dioxide quality is very bad, sulfur dioxide quality is relatively good, carbon monoxide quality is good, and air particles quality is also bad, Then air quality is highly unhealthy.

9 If ozone quality is good, nitrogen dioxide quality is relatively good, sulfur dioxide quality is good, carbon monoxide quality is good, and air particles quality is also bad, Then air quality is unhealthy

10 If ozone quality is very bad, nitrogen dioxide quality is dangerous, sulfur dioxide quality is very bad, carbon monoxide quality is good, and air particles quality is also good, Then air quality is dangerous.

11 If ozone quality is dangerous, nitrogen dioxide quality is dangerous, sulfur dioxide quality is dangerous, carbon monoxide quality is dangerous, and air particles quality is good, Then air quality is dangerous.

12 If ozone quality is dangerous, nitrogen dioxide quality is dangerous, sulfur dioxide quality is dangerous, carbon monoxide quality is dangerous, and air particles quality is relatively good, Then air quality is dangerous.

13 If ozone quality is relatively good, nitrogen dioxide quality is relatively good, sulfur dioxide quality is relatively good, carbon monoxide quality is relatively good, and air particles quality is also relatively good, Then air quality is regular.

14 If ozone quality is relatively bad, nitrogen dioxide quality is relatively bad, sulfur dioxide quality is relatively bad, carbon monoxide quality is relatively bad, and air particles quality is also relatively bad, Then air quality is unhealthy.

15 If ozone quality is bad, nitrogen dioxide quality is bad, sulfur dioxide quality is bad, carbon monoxide quality is bad, and air particles quality is also relatively bad, Then air quality is unhealthy.

16 If ozone quality is dangerous, nitrogen dioxide quality is dangerous sulfur dioxide quality is dangerous, carbon monoxide quality is dangerous, and air particles quality is also dangerous, Then air quality is dangerous.

17 If ozone quality is very bad, nitrogen dioxide quality is very bad, sulfur dioxide quality is very bad, carbon monoxide quality is very bad, and air particles quality is also very bad, Then air quality is highly unhealthy.

18 If ozone quality is good, nitrogen dioxide quality is relatively good, sulfur dioxide quality is relatively bad, carbon monoxide quality is bad, and air particles quality is also very bad, Then air quality is unhealthy.

19 If ozone quality is dangerous, nitrogen dioxide quality is very bad, sulfur dioxide quality is bad, carbon monoxide quality is relatively bad, and air particles quality is also relatively good, Then air quality is highly unhealthy.

20 If ozone quality is good, nitrogen dioxide quality is relatively good, sulfur dioxide quality is good, carbon monoxide quality is relatively good, and air particles quality is also good, Then air quality is regular.

21 If ozone quality is relatively bad, nitrogen dioxide quality is good, sulfur dioxide quality is relatively bad, carbon monoxide quality is good, and air particles quality is also relatively bad, Then air quality is unhealthy for the allergic population.

22 If ozone quality is bad, nitrogen dioxide quality is relatively good, sulfur dioxide quality is bad, carbon monoxide quality is relatively good, and air particles quality is also bad, Then air quality is unhealthy.

23 If ozone quality is good, nitrogen dioxide quality is bad, sulfur dioxide quality is good, carbon monoxide quality is bad, and air particles quality is also good, Then air quality is unhealthy.

24 If ozone quality is good, nitrogen dioxide quality is very bad, sulfur dioxide quality is good, carbon monoxide quality is very bad, and air particles quality is also good, Then air quality is highly unhealthy.

25 If ozone quality is dangerous, nitrogen dioxide quality is good, sulfur dioxide quality is dangerous, carbon monoxide quality is good, and air particles quality is also dangerous, Then air quality is dangerous.

For example, the 6 diagrams in the first row in Fig. 8 indicate the assumptions and conclusions of the 
first rule. Additionally, each column in this diagram exhibits a certain factor. Like demonstrated in Fig. 8 , each of the 5 yellow columns (at the left side), are respectively from left to right associated with one of the 5 factors: Ozone quality, nitrogen dioxide quality, sulfur dioxide quality, carbon monoxide quality, and particles quality. Each rule's number has been indicated at the left side of diagram. The 5 left columns in the figure display the membership functions of assumptions (if-section). The 6th column indicates the membership functions of the rule (then-section). The empty diagrams demonstrate that the rule in question has had no factor.

The factors and their current values are shown on top of each column. Red lines present the average of marks given to each factor. The blue column on the right side indicates the result of applying each fuzzy rule to each fuzzy set defined for that rule, according to the given marks. In this example, ozone level of 37.5, with nitrogen dioxide level of 67.5, sulfur dioxide level of 67.5, carbon monoxide level of 37.5 and air particles 37.5 , are associated with air quality of 67.5. This quality level means unhealthy air for allergic population. It is noteworthy that "Centroid Method" of Matlab Software has been used for defuzzifying. In the end, with the help of menu, the two-by-two surface of inputs can be arranged with the outputs. The following equation indicates that sulfur dioxide and ozone, compared to the output level, are beyond the range of 242 .

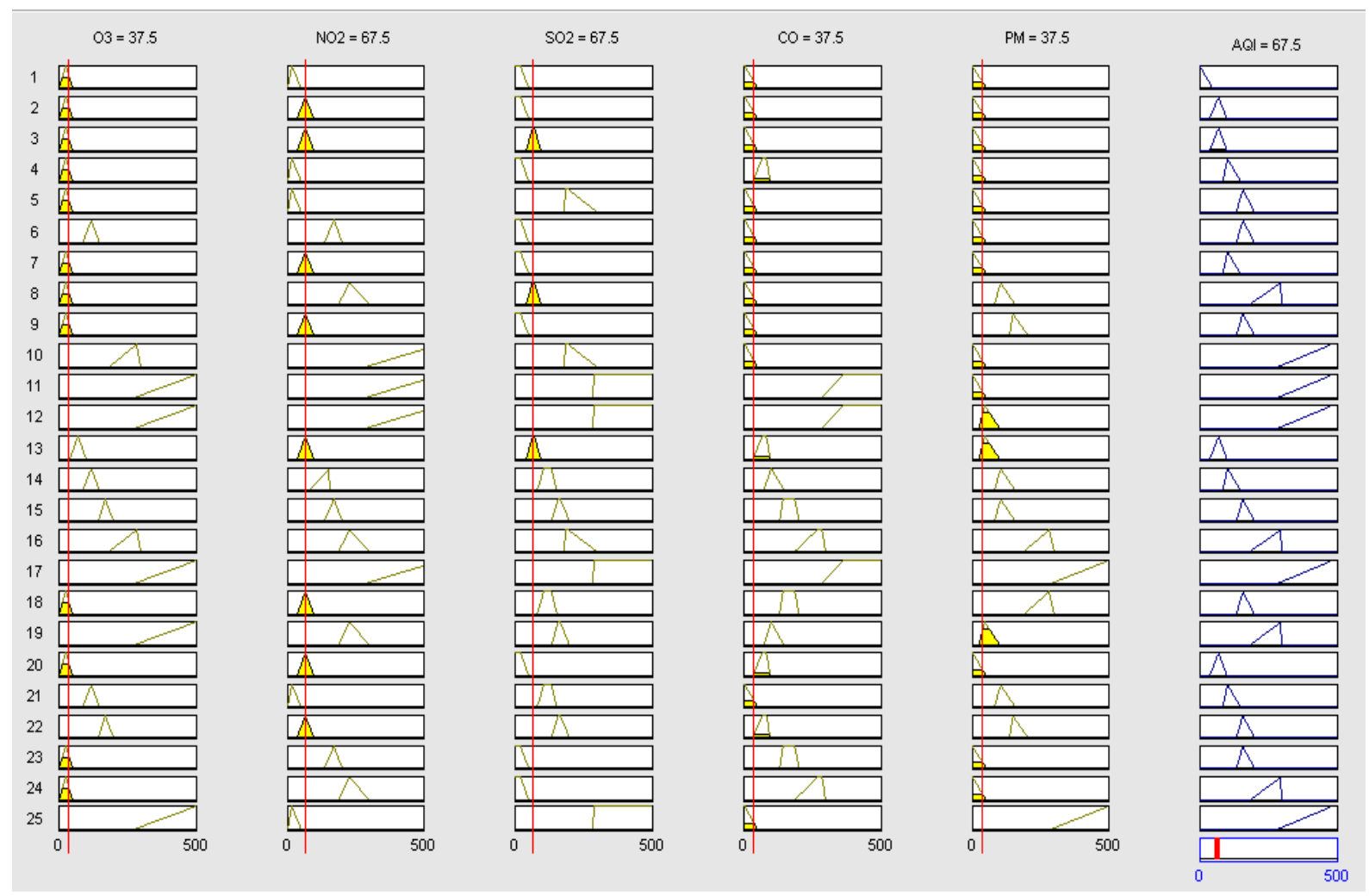

Fig. 8. The rules of fuzzy sets

\section{Conclusions}

In addition to providing sets of influential factors in Tehran's Air Quality Index, this study has aimed to develop the associated fuzzy inferential system to be applied for measuring the quality of respiratory air. The indexes are divided into 5 general categories: Ozone quality, nitrogen dioxide quality, sulfur dioxide quality, carbon monoxide quality, and the quality of air particles. According to the 5 mentioned sets of factors, fuzzy sets and membership functions of each adjacent fuzzy set has been determined. In the end, the fuzzy inferential system for measuring air pollution quality has been presented. The associated diagrams were drawn with Matlab software (Guide, 1998; Jang, 1997). 


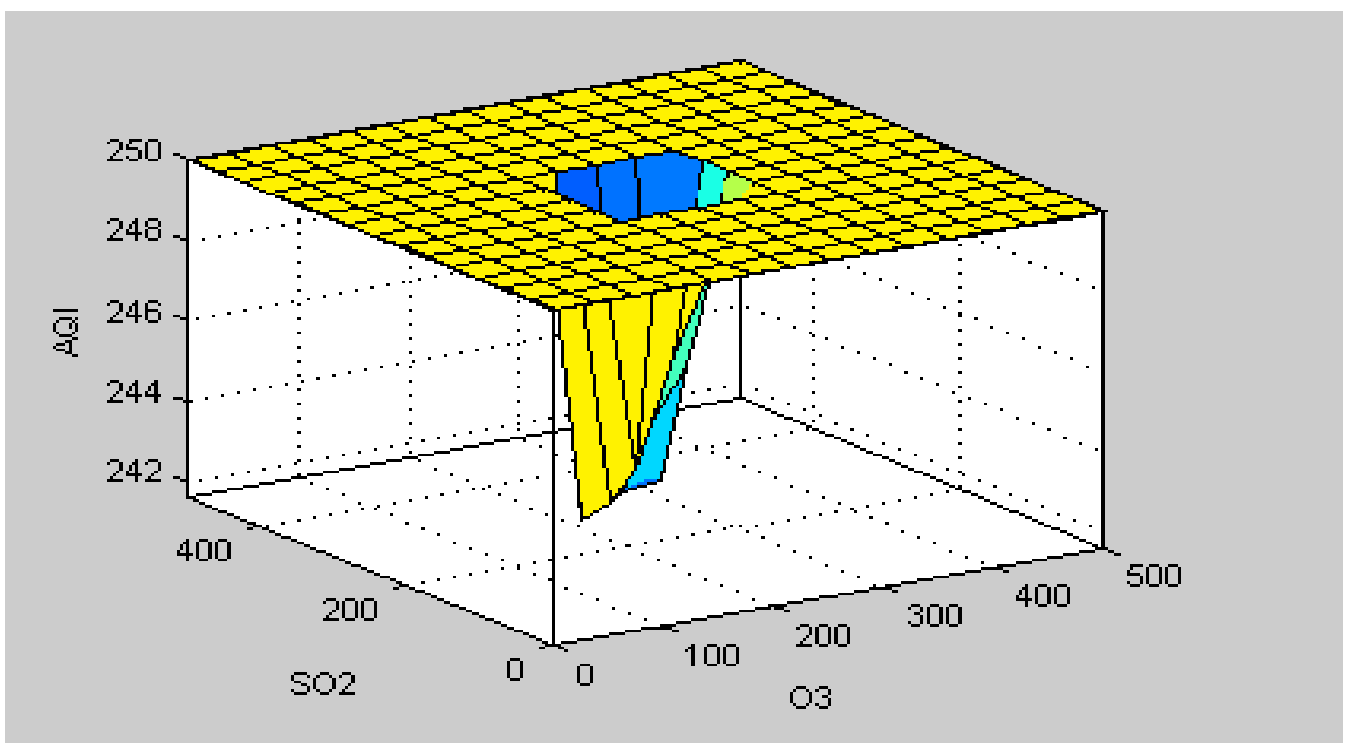

Fig. 9. The relationship between $\mathrm{O} 3$ and $\mathrm{So} 2$

\section{Acknowledgement}

The authors would like to thank the anonymous referees for constructive comments on earlier version of this paper.

\section{References}

Guide, M. U. S. (1998). The mathworks. Inc., Natick, MA, 5.

Gurjar, B. R., Butler, T. M., Lawrence, M. G., \& Lelieveld, J. (2008). Evaluation of emissions and air quality in megacities. Atmospheric Environment, 42(7), 1593-1606.

Jang, J. R. (1997). MATLAB: Fuzzy Logic Toolbox User's Guide: Version 1. Math Works.

Kyrkilis, G., Chaloulakou, A., \& Kassomenos, P. A. (2007). Development of an aggregate Air Quality Index for an urban Mediterranean agglomeration: Relation to potential health effects. Environment International, 33(5), 670-676.

Mamdani, E. H. (1974, December). Application of fuzzy algorithms for control of simple dynamic plant. In Proceedings of the Institution of Electrical Engineers (Vol. 121, No. 12, pp. 1585-1588). IET Digital Library.

Mamdani, E. H., \& Assilian, S. (1975). An experiment in linguistic synthesis with a fuzzy logic controller. International journal of man-machine studies, 7(1), 1-13.

Mamdani, E. H. (1976). Advances in the linguistic synthesis of fuzzy controllers. International Journal of Man-Machine Studies, 8(6), 669-678.

Mamdani, E. H. (1977). Application of fuzzy logic to approximate reasoning using linguistic synthesis. Computers, IEEE Transactions on, 100(12), 1182-1191.

Tehran's Air Quality Report (2013), Tehran's Air Quality Control Bureau an affiliate of municipality.

Zadeh, L. A. (1965). Fuzzy sets. Information and control, 8(3), 338-353.

Zadeh, L. A. (1968). Probability measures of fuzzy events. Journal of mathematical analysis and applications, 23(2), 421-427.

Zadeh, L. A. (1997). Toward a theory of fuzzy information granulation and its centrality in human reasoning and fuzzy logic. Fuzzy sets and systems, 90(2), 111-127.

Zadeh, L. A. (1982). A note on prototype theory and fuzzy sets. Cognition,12(3), 291-297.

Zadeh, L. A. (1983). A computational approach to fuzzy quantifiers in natural languages. Computers 
\& Mathematics with Applications, 9(1), 149-184.

Zadeh, L. A. (1996). Fuzzy logic= computing with words. Fuzzy Systems, IEEE Transactions on, 4(2), 103-111.

Zimmermann, H. J. (1992). Fuzzy Set Theory and Its Applications Second, Revised Edition. Kluwer academic publishers. 